\title{
Safety and Efficacy of 2 Intracavernous Injections of Allogeneic Wharton's Jelly-Derived Mesenchymal Stem Cells in Diabetic Patients with Erectile Dysfunction: Phase 1/2 Clinical Trial
}

\author{
Saddam Al Demour ${ }^{a}$ Sofia Adwan ${ }^{\text {b, c }}$ Hanan Jafar ${ }^{b, d}$ Reem Rahmeh ${ }^{b}$ \\ Hussam Alhawari ${ }^{\mathrm{e}}$ Abdalla Awidi ${ }^{\mathrm{b}, \mathrm{e}}$ \\ aDivision of Urology, Department of Special Surgery, School of Medicine, The University of Jordan, Amman, Jordan; \\ ${ }^{b}$ Cell Therapy Center, The University of Jordan, Amman, Jordan; 'Department of Medical Laboratory Sciences, \\ Faculty of Allied Medical Sciences, Al-Ahliyya Amman University, Amman, Jordan; ${ }^{\mathrm{d} D e p a r t m e n t}$ of Anatomy and \\ Histology, School of Medicine, The University of Jordan, Amman, Jordan; 'Department of Medicine, School of \\ Medicine, The University of Jordan, Amman, Jordan
}

\section{Keywords}

Erectile dysfunction · Diabetes mellitus · Stem cells · Safety · Efficacy

\begin{abstract}
Background and Objectives: Stem cell therapy is a novel treatment with regenerative ability that can treat erectile dysfunction (ED). This phase $1 / 2$ clinical trial (NCT02945449) using 2 consecutive intracavernous (IC) injections of allogeneic Wharton's jelly-derived mesenchymal stem cells (WJMSCs) was studied for the first time in the treatment of diabetic patients with ED. The primary outcome was to assess the safety and tolerability, and the secondary outcome was to assess the efficacy of 2 consecutive IC injections of allogeneic WJ-MSCs in diabetic ED. Patients and Methods: Twenty-two diabetic patients with refractory ED were included. Two consecutive IC injections of allogeneic WJ-MSCs were performed. Tolerability was assessed immediately, and at 24 h, safety was evaluated for 12 months. Efficacy was assessed using International Index of Erectile Function-5 (IIEF-5), Erection Hardness Score (EHS), and Color Duplex Doppler Ultrasound for 12 months. Results: The procedure was well-tolerated. Minimal and transient adverse events were redness
\end{abstract}

karger@karger.com

(c) 2021 S. Karger AG, Basel

www.karger.com/uin

Karger and bruising at the site of injections. There were no patientreported serious adverse effects. There were significant improvements in IIEF-5, EHS, peak systolic velocity (PSV) basal, and 20-min PSV, all over the follow-up time points in comparison to the baseline. Conclusion: This is the first human study with proven tolerability, safety, and efficacy of IC injections of allogeneic WJ-MSCs for the treatment of diabetic patients with ED.

(c) 2021 S. Karger AG, Basel

\section{Introduction}

Erectile dysfunction (ED) has a severe negative impact on the quality of life and can be a consequence of underlying psychogenic and organic diseases affecting erectile tissues [1]. Various risk factors contribute to the incidence of organic ED, including diabetes mellitus (DM), cardiovascular disease, age, smoking, atherosclerosis, metabolic syndrome, hypertension, hyperlipidemia, and obesity [2].

The prevalence of DM in the United States was 8.3\% in the total population in 2010, and $26.9 \%$ in patients aged 65 years or older [3]. ED is a common complication of 
DM. In men with DM, the risk of developing ED is 3 times higher than in nondiabetic men, with a prevalence of 50 $75 \%$, and phosphodiesterase-5 (PDE5) inhibitors are less efficacious in this patient group [4-6].

The pathophysiology of diabetic ED is multifactorial, with neurogenic, endothelial, and/or smooth muscle dysfunction as the main factors [7]. Long-standing DM leads to apoptosis and dysfunction of the corporal endothelium and smooth muscle, and diminishes the amount of NOreleasing penile nerves, resulting in increased oxidative stress in cavernous tissues $[8,9]$.

Currently, most patients with ED can be treated successfully with PDE5 inhibitors. However, diabetic men with ED usually do not respond well to PDE5 inhibitors [10]. Therefore, more effective and lasting treatment options for DM patients with ED are desirable.

Stem cell-based therapies have received attention regarding their potential for erectile function (EF) recovery and structural tissue repair in diabetic ED patients [11]. Mesenchymal stem cells (MSCs) are multipotent SCs that can be retrieved from the bone marrow, adipose tissue, umbilical cord, placenta, liver, muscle, and dental pulp [12]. Preclinical studies have shown that treatment with MSCs significantly improves EF recovery in diabetic animal models with ED [13]. This EF improvement appears to be due to the paracrine factors secreted by the injected cells, namely, cytoprotective, anti-fibrotic, and antiapoptotic molecules [14].

The human umbilical cord, which consists largely of Wharton's jelly (WJ), has been characterized as a promising source of MSCs [15]. Wharton's jelly-derived MSCs (WJMSCs) can be isolated from the WJ intervascular, perivascular, and subamniotic areas [16]. WJ-MSCs are better sources for MSCs than bone marrow or adipose tissue because they are readily available and there are no serious ethical issues involved, since the umbilical cords are usually discarded. Also, WJ-MSCs have a high proliferation rate and do not carry any teratogenic or carcinogenic risks when transplanted [17]. In comparison, MSCs from the bone marrow or fatty tissue have several disadvantages. First, only a small portion $(0.05-0.001 \%)$ of bone marrow cells are MSCs [18]. Second, bone marrow biopsy is an invasive and painful procedure. Although adipose tissue is abundant, its gathering requires an invasive procedure [19].

To the best of our knowledge, this is the first clinical trial using 2 consecutive intracavernous (IC) injections of allogeneic WJ-MSCs in the treatment of diabetic patients with ED. As the primary outcome, the safety of this therapeutic approach was assessed; as the secondary outcome, its efficacy was evaluated.

\section{Methods}

Study Design and Approval

This study is a prospective phase $1 / 2$, open-label, single-arm, and single-center trial. The study was approved by the Institutional Review Board of Cell Therapy Center, the University of Jordan, Amman, Jordan (reference number: IRB/4/2017), and prospectively registered on clinicaltrials.gov (NCT02945449). All participants were informed regarding the study design and were educated about IC injections of allogeneic WJ-MSCs, questionnaires used in the study, and Color Doppler Duplex Ultrasound (CDDU) of penile hemodynamics. Participants signed a written informed consent before participation in the study following the Helsinki Declaration.

\section{Inclusion and Exclusion Criteria}

To be selected for this study, participants had to meet all the following criteria: adult male patients with age ranging from 25 to 75 years, type 1 or type 2 diabetes with an $\mathrm{HbAlc} \leq 10 \%$, history of diabetes $\geq 5$ years, body mass index between 20 and 30, baseline International Index of EF (IIEF-5) score of $<22$, and history of chronic ED for at least 6 months with proven unresponsiveness to previous medical therapies such as PDE5-inhibitors and prostaglandin E1.

Patients with any of the following conditions were excluded: clinically evident penile anatomical deformities (e.g., Peyronie's disease); history of priapism, skin irritation, infection, or wound in the immediate areas of skin entry for penile injection; bleeding or clotting disorders; use of anticoagulant therapy; current urinary tract infection; current or previous infection with human immunodeficiency or hepatitis viruses; any previous penile implant; penile vascular surgery or radical prostatectomy; current or previous malignancy; prostate-specific antigen $(>4 \mathrm{ng} / \mathrm{mL})$; untreated hypogonadism or low serum total testosterone $(<200 \mathrm{ng} / \mathrm{dL})$; uncontrolled hypertension or hypotension (systolic blood pressure $>170$ or $<90$ $\mathrm{mm} \mathrm{Hg}$, and diastolic blood pressure $>100$ or $<50 \mathrm{~mm} \mathrm{Hg}$ ); reported unstable cardiovascular disease (e.g., unstable angina, myocardial infarction within past 6 months, cardiac failure or life-threatening arrhythmia, and congestive heart failure) or symptomatic postural hypotension within 6 months before screening; systemic autoimmune disorder; significant active systemic or localized infection; and immunosuppressive medication. The following laboratory screening results also had to be normal: luteinizing hormone, testosterone and prolactin, liver function tests (ALT, AST, and GGT), kidney function tests and/or electrolytes (urea, creatinine, $\mathrm{Na}, \mathrm{K}$, and $\mathrm{Ca}$ ), complete blood count with differential, coagulation profile (INR, PT, and PTT), lipid profile (HDL, LDL, TG, and total cholesterol), urinalysis and culture, hepatitis B and C (HBs Ag and hepatitis $\mathrm{C}$ antibodies), human immune deficiency, and lues serology (VDRL). Patients whose compliance to continue throughout the trial seemed insufficient were also excluded.

\section{Cell Isolation, Culture, and Expansion}

Master Cell Bank

Informed consent was acquired from all donors. WJ was collected from blood group O Rh-negative healthy donors, full-term women, who underwent an elective cesarean section. Samples were processed within $2-4 \mathrm{~h}$ of collection. The WJ was transferred to the laboratory, where it was disinfected and cut into $0.5-1 \mathrm{~mm}^{2}$ pieces. These pieces were then transferred to $150 \mathrm{~cm}^{2}$ plates containing Minimum Essential Medium Eagle -Alpha Modification (Gibco, UK) supplemented with $5 \%$ platelet lysate, $1 \%$ penicillin- 
streptomycin (Gibco, USA), 3U heparin (Innohep; LEO Pharma, Ballerup, Denmark), and 4 mML-glutamine. Cells were then incubated in a humidified atmosphere containing $5 \% \mathrm{CO}_{2}$ at $37^{\circ} \mathrm{C}$. The medium was replaced after 6 days to allow cells to migrate from the explants. Adherent confluent cells were harvested with TrypLE 10X (Gibco, Germany) and propagated at a seeding density of 4,000 cells $/ \mathrm{cm}^{2}$. For the master bank preparation, first and third passage cells were cryopreserved in freezing bags with synth-afreeze (Gibco, Germany). Cells at passage 4 were used for the injections.

\section{MSC Characterization}

Expanded MSCs were phenotypically tested for MSC markers. Briefly, a total of $0.25 \times 10^{5}$ cells were resuspended in $0.5 \mathrm{~mL}$ phosphate-buffered saline (Gibco, USA) and incubated with the antibodies of BD Stemflow ${ }^{\mathrm{TM}}$ hMSC Analysis Kit (BD Biosciences, USA) for $20 \mathrm{~min}$ at room temperature. The fluorescence intensity of the cells was evaluated by flow cytometry (BD FACSCanto II; BD Biosciences, USA).

Additionally, MSCs were tested for multilineage differentiation potential. For osteogenic and adipogenic differentiation, cells were cultured in StemPro osteogenesis differentiation media and StemPro adipogenesis differentiation media (ThermoFisher, USA), respectively, as per the manufacturer's recommendation. Following osteogenic induction, cultures were stained using Alizarin Red (Allied Signal, Germany), and following adipogenic differentiation, cultures were stained using Oil Red O (Sigma-Aldrich, USA) to assess extracellular deposits.

\section{Release Tests}

Released viable MSCs were tested for mycoplasma, endotoxin, and microbial contamination. Furthermore, cells were fully karyotyped.

\section{Viability Test}

Viability was assessed by trypan blue exclusion dye assay. Cells were counted both using an automated cell counter (Countess; Thermo, USA) and manually using a hematocytometer. Viable cells were presented as a percent of the total. The acceptable viability percentage was set at $80 \%$.

Mycoplasma, Endotoxin, and Bacterial Contamination Tests

The absence of mycoplasma contamination was verified using the MycoSEQ ${ }^{\mathrm{TM}}$ Mycoplasma Detection Kit with Discriminatory Positive Control (Invitrogen ThermoFisher, USA). According to the manufacturer's instructions, screening and detecting mycoplasma in cells and culture medium were performed. The culture medium was cultured on blood, MacConkey, and chocolate agar plates. Blood and MacConkey agar plates were incubated at $37^{\circ} \mathrm{C}$ for $72 \mathrm{~h}$. Chocolate agar was placed in an anaerobic jar and incubated at $37^{\circ} \mathrm{C}$ for $72 \mathrm{~h}$. After $72 \mathrm{~h}$ of incubation, culture results were released as negative if no colonies were seen on all plates. Limulus amebocyte lysate QCL-1000 (Lonza, Switzerland) test was used to detect Gram-negative bacterial endotoxin before cells were injected. The limulus amebocyte lysate test was performed according to the manufacturer's instructions.

\section{Cytogenetic Test}

Karyotype analysis for passage 3 MSCs was carried out to eliminate the possibility of chromosomal abnormalities. Twenty differ- ent metaphase spreads of cells were treated with colchicine and were harvested for fixation with a hypotonic solution. Fixed cells were then dropped onto a slide, dried, and stained to observe a G banding pattern.

\section{Patient Assessment and Clinical Procedure}

At baseline, patients were requested to fill in validated IIEF-5 and Erection Hardness Score (EHS) questionnaires. All patients underwent a thorough medical and sexual history, complete physical examination, and laboratory investigations as described above.

Eligible participants underwent penile CCDU as baseline assessment of penile hemodynamics by measuring peak systolic velocity (PSV), end-diastolic velocity (EDV), both measured in centimeter per second, and the resistive index (RI) in both cavernosal vessels before (basal PSV, EDV, and RI) and $20 \mathrm{~min}$ after an IC injection of $20 \mu \mathrm{g}$ of alprostadil (20-min PSV, EDV, and RI). After that, eligible participants received 2 IC ex vivo expanded allogeneic WJ-MSC injections with a 30-day interval between both injections. Each time, passage 2 or passage 3 cells were used for the injections; these were suspended at a density of $20 \times 10^{6}$ cells $/ 4 \mathrm{~mL}$ normal saline and loaded into $1 \mathrm{~mL}$ sterile syringes. Four IC injections were given at each time at 4 sites: 1 proximal and 1 distal injection into each corpus cavernosum. Patients were instructed not to take any medications to treat ED for the study's whole duration.

\section{Outcome Measures}

Tolerability was assessed by observing and questioning patients for pain during IC injections; pain intensity was assessed on a $0-10$ visual analog scale (VAS), in which $0-3$ represented mild pain, 4-6 represented moderate pain, and 7-10 represented severe pain. Safety outcomes were assessed immediately, at $24 \mathrm{~h}, 1,3,6$, and 12 months after IC injections.

Safety was assessed by examination of the injection sites for bleeding, bruising, tenderness, swelling, hypo- and hyperthermia, erythema, urticaria, or indurations, by measuring the vital signs (temperature, heart rate, respiratory rate, and blood pressure) and by interviewing patients for any other potential adverse events that might have occurred. Specifically, patients were asked at visit about nervous system symptoms (headache, dizziness, and loss of consciousness), cardiovascular symptoms (chest pain, palpitation, shortness of breath, fainting, ankle edema, weakness, and fatigue), respiratory symptoms (cough, nasal discharge, and fever), gastrointestinal symptoms (abdominal pain, nausea, vomiting, bloating, and diarrhea), and urinary tract symptoms (hematuria, frequency, urgency, poor flow, and burning sensation on micturition). Moreover, the same laboratory tests taken at baseline were repeated at 1 and 3 months during follow-up.

Efficacy of the IC injections of allogeneic WJ-MSCs was assessed qualitatively by IIEF-5 and EHS questionnaires at 1, 3, 6, and 12 months of follow-up. Moreover, the follow-up with penile CDDU was performed 3 months after the second IC injection.

\section{Statistical Analysis}

Data were reported as mean \pm SD. Efficacy outcomes were analyzed by Friedman's test for multiple nonparametric comparisons, and comparisons at each point of the follow-up with baseline were made using Wilcoxon signed-rank test. A statistical significance level of $5 \%$ and a 2 -tailed test were used. Statistical analyses were performed using IBM SPSS Statistics for Windows, version 20 (IBM Corp., Armonk, NY, USA). 


\section{Results}

\section{Characterization of WJ-MSCs}

The released WJ-MSCs exhibited the typical fibroblast-like spindle-shaped morphology (Fig. 1). These cells were also positive for the expression of the International Society for Cellular Therapy (ISCT) validated markers (CD90, CD105, CD73, and CD44) and were negative for CD34, CD45, CD11b, CD19, and HLA-DR (Fig. 2). Furthermore, upon induction, WJ-MSCs were able to differentiate toward adipogenic and osteogenic lineages (Fig. 3a and b, respectively). WJ-MSCs presented a normal chromosome karyotype without polyploidy. All cultures were free of mycoplasma, bacterial, and endotoxin contamination.

\section{Patient Population}

A total of 32 patients were enrolled in this clinical trial between September 2018 and August 2019. Seven patients were excluded from the study as they did not meet the inclusion criteria, 3 patients refused to participate, and 1 patient missed follow-up. A total of 22 patients underwent final analysis. The mean age of participants was $59.18 \pm 8.45$ years. The baseline patients' characteristics are shown in Table 1 .

\section{Primary Outcomes}

The primary outcomes were safety and tolerability. The procedure was well-tolerated and safe, and no patient reported serious adverse effects. Ten patients reported mild pain at the injection site only during the procedure; the VAS pain score ranged from 0 to 3 . On observing the injection sites during follow-up, the majority of patients had no pain, bleeding, erythema, urticaria, bruising, swelling, priapism, hypothermia, or hyperthermia. Two patients experienced minimal redness and swelling at the base of the penis and bruises at the distal shaft of the penis $24 \mathrm{~h}$ after the first injection, which was managed conservatively and resolved within 1 week. However, 3 months after the second injection, 1 patient reported a very small fibrous plaque (Peyronie's disease) on the dorsal aspect of the penis without curvature which did not interfere with sexual intercourse. Vital signs (body temperature, heart rate, respiratory rate, and blood pressure) were normal in all patients at all follow-up visits. Additionally, there were no systemic adverse events detected during follow-up, including cardiovascular, respiratory, urinary, nervous, and gastrointestinal systems. Furthermore, standard biochemistry and hematology laboratory tests showed no abnormal results at 1 month after the first IC injection and

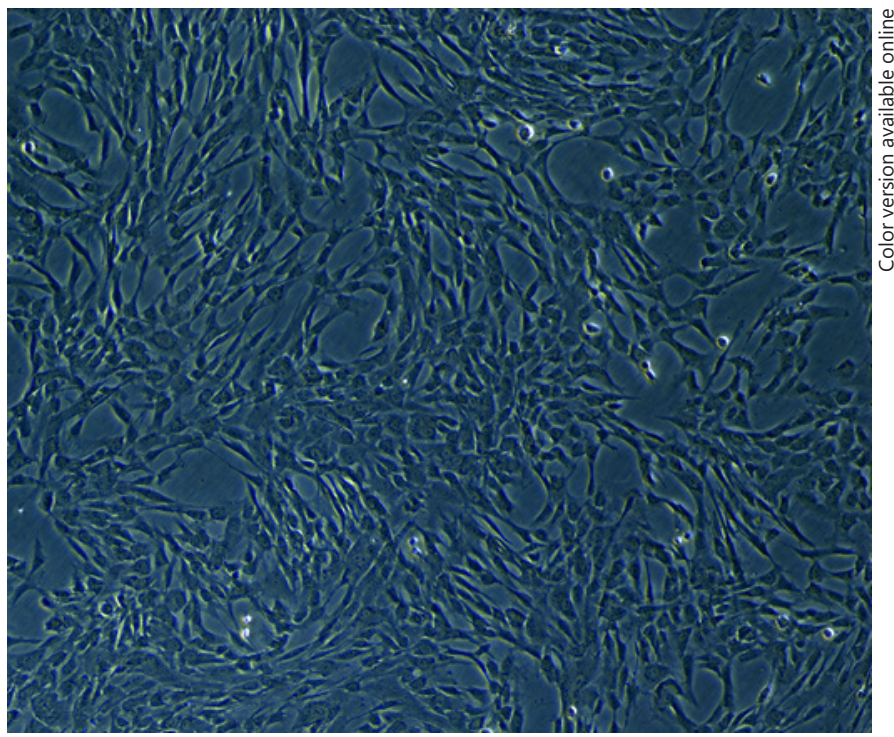

Fig. 1. WJ-derived MSCs. Representative image shows the light microscopic view of WJ-MSCs at P4 before injection. WJ, Wharton's jelly; MSCs, mesenchymal stem cells.

Table 1. Baseline patients' characteristics $(n=22)$

\begin{tabular}{lc}
\hline Age, years, mean \pm SD & $59.18 \pm 8.45$ \\
BMI, kg/m ${ }^{2}$ & $23.8 \pm 2.5$ \\
Smoking, $n(\%)$ & $8(35)$ \\
DM duration, years & $11.06 \pm 5.45$ \\
Comorbidities, \% & 39.1 \\
\hline
\end{tabular}

BMI, body mass index; DM, diabetes mellitus; SD, standard deviation.

3 months after the second IC injection (see online suppl. Table 1; see www.karger.com/doi/10.1159/000517364 for all online suppl. material).

\section{Secondary Outcome}

Overall, the mean IIEF-5 scores significantly improved at all follow-up time points compared to baseline. There was a remarkable and significant improvement of IIEF-5 scores at 1 month after the IC injections compared to baseline $(12.3 \pm 3.2$ vs. $11.5 \pm 2.7, p=0.002)$. Also, 3 months after the injections, there was a further increase in scores which was also significantly different from baseline scores (15.6 \pm 3.4 vs. $11.5 \pm 2.7, p<0.0001)$. After 6 months, the IIEF- 5 scores reached maximum improvement compared to baseline (16.9 \pm 4 vs. $11.5 \pm 2.7, p<$ $0.0001)$. However, at 12 months, there was a significant 


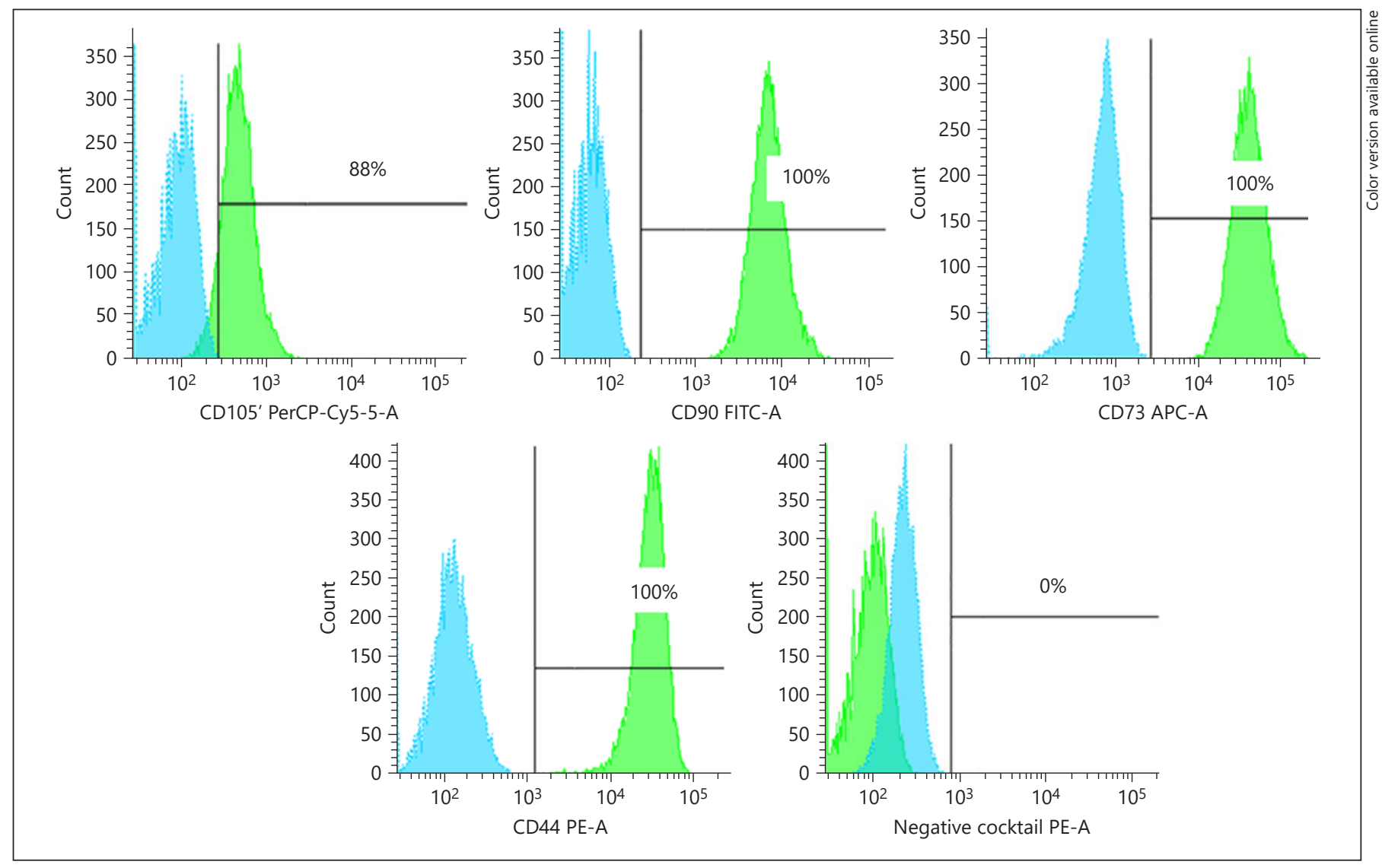

Fig. 2. Representative image of the immunophenotypic characterization of MSCs: positive expression of CD90, CD105, CD73, and CD44, and the negative cocktail: negative expression of hematopoietic markers: CD34, CD45, CD11b, CD19, and HLA-DR. MSCs, mesenchymal stem cells.
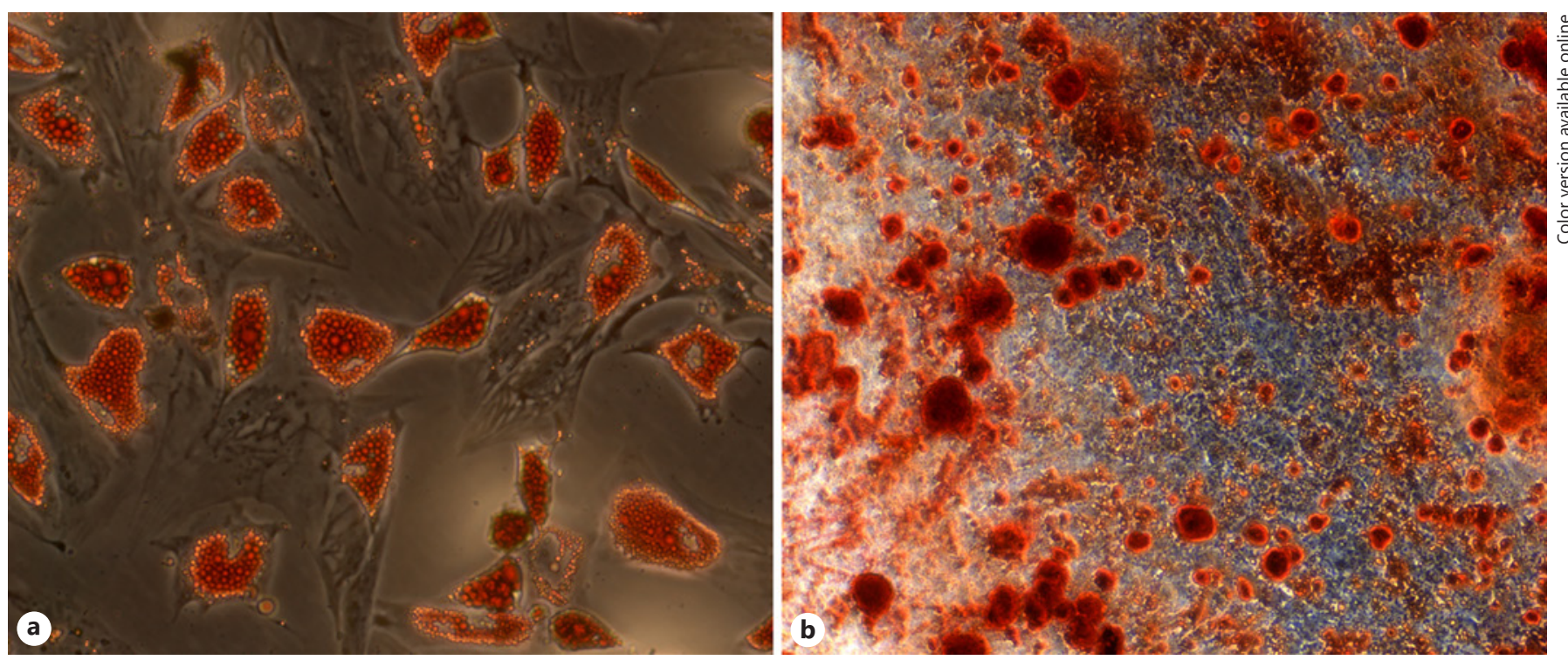

Fig. 3. In vitro differentiation potential of MSCs. Representative images of MSC differentiation potential after induction toward adipogenic (a) and osteogenic lineages (b). MSCs, mesenchymal stem cells. 
Fig. 4. Changes in IIEF-5 and EHS after IC injection of WJ-MSCs at different time points. MSCs, mesenchymal stem cells; IC, intracavernous; WJ, Wharton's jelly; IIEF5, International Index of Erectile Function; EHS, Erection Hardness Score.

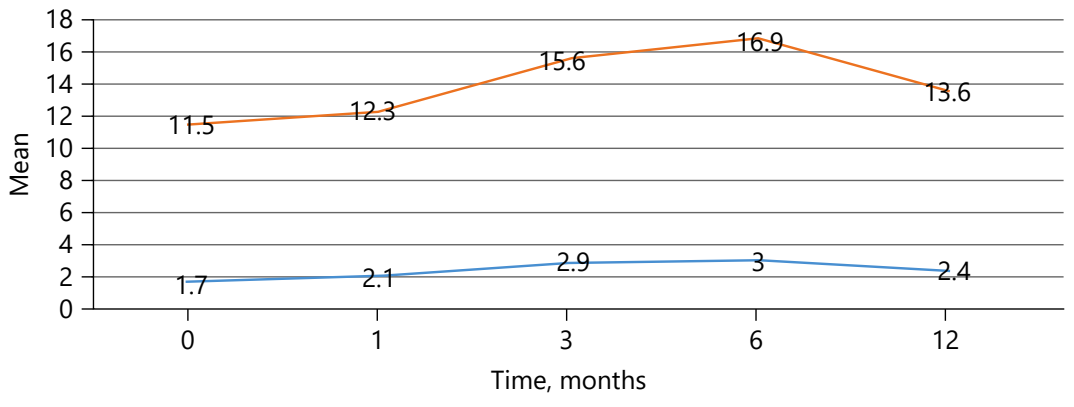

Table 2. Changes in IIEF-5 and EHS after IC injection of WJ-MSCs at different time points

\begin{tabular}{lrrlrlrrrr}
\hline & Baseline & 1 month & $p$ value & 3 months & $p$ value & 6 months & $p$ value & 12 months & $p$ value \\
\hline IIEF-5, mean \pm SD & $11.5 \pm 2.7$ & $12.3 \pm 3.2$ & 0.002 & $15.6 \pm 3.4$ & $<0.0001$ & $16.9 \pm 4$ & $<0.0001$ & $13.6 \pm 4.2$ \\
EHS, mean \pm SD & $1.7 \pm 0.7$ & $2.1 \pm 0.8$ & 0.0039 & $2.9 \pm 0.7$ & $<0.0001$ & $3 \pm 0.8$ & $<0.0001$ & $2.4 \pm 0.7$ & $<0.0002$ \\
\hline
\end{tabular}

WJ, Wharton's jelly; MSCs, mesenchymal stem cells; IC, intracavernous; SD, standard deviation; IIEF-5, international index of erectile function; EHS, Erection Hardness Score.

Table 3. CDDU before and after IC injections of WJ-MSCs

\begin{tabular}{lccc}
\hline & $\begin{array}{l}\text { Baseline } \\
(\text { mean } \pm \text { SD })\end{array}$ & $\begin{array}{l}\text { Three months } \\
(\text { mean } \pm \text { SD })\end{array}$ & $p$ value \\
\hline Basal PSV & $12.06 \pm 10.33$ & $16.35 \pm 12.71$ & $\mathbf{0 . 0 3 3 2}$ \\
Basal EDV & 0 & 0 & - \\
Basal RI & $0.63 \pm 0.46$ & $0.67 \pm 0.44$ & 0.6719 \\
20-min PSV & $38.34 \pm 12.93$ & $48.72 \pm 17.05$ & $<\mathbf{0 . 0 0 0 1}$ \\
20-min EDV & $4.98 \pm 3.74$ & $5.79 \pm 3.88$ & 0.2579 \\
20-min RI & $0.85 \pm 0.1$ & $0.83 \pm 0.1$ & 0.9098 \\
\hline
\end{tabular}

PSV, peak systolic velocity; EDV, end-diastolic velocity; RI, resistive index; WJ, Wharton's jelly; MSCs, mesenchymal stem cells; IC, intracavernous; SD, standard deviation; CDDU, Penile Color Duplex Doppler Ultrasound.

decline in the score compared to the 6-month values (13.6 \pm 4.2 vs. $16.9 \pm 4, p<0.0001$ ) (Table 2 ; Fig. 4 ).

The EHS score also significantly improved over time. At baseline, the mean score was $1.7 \pm 0.7$. It reached the maximum score at 6 months after IC injections $(3 \pm 0.8)$ $(p<0.0001)$. There was also a drop in the mean score at 12 months compared to the 6-month value, which was not significant $(2.4 \pm 0.7$ vs. $3 \pm 0.8)$ but was still higher than at baseline (Table 2; Fig. 4).
The mean basal PSV was significantly higher 3 months after the injections $(12.06 \pm 10.33$ vs. $16.35 \pm 12.71, p=$ 0.0332 ). Also, the 20 -min PSV after IC injections was significantly higher than at baseline $(38.34 \pm 12.93$ vs. 48.72 $\pm 17.05, p<0.0001)$ (Table 3).

\section{Discussion}

This study is the first clinical trial using 2 consecutive IC injections of allogeneic WJ-MSCs to evaluate longterm safety and efficacy in treating diabetic patients with ED. In recent years, SC-based therapy gained much attention in medicine and specifically in urology for treatment of ED from various etiological factors [20,21]. Recently, the connective tissue WJ of the human umbilical cord is considered a potential alternative tissue source of MSCs [22]. WJ possesses desirable characteristics such as a large, rapidly available donor pool; a noninvasive and painless collection procedure; and an ethically noncontroversial source of MSCs [23].

In our study, the treatment was well-tolerated and safe. It was also effective in improving $\mathrm{EF}$ in diabetic patients followed up for 12 months. We did not observe any serious adverse events except for mild pain during injection and a minimal local effect at the injection site that disap- 
peared without a negative impact on the patient's activity. One patient reported a small fibrous plaque on the dorsal aspect of the penis, and we attributed this plaque formation due to an excessive use of IC injection therapy (alprostadil $20 \mu \mathrm{g} / \mathrm{mL}$ ) for 2 years before participation in this trial. Importantly, the majority of patients reported significant improvement in EF and EHS. Most of the patients reported an improvement at all time points during follow-up. The maximum improvement was reported after 6 months when all participants had received 2 injections.

The improvement in EF was shown by validated questionnaires (IIEF-5 and EHS) and also shown more objectively on CDDU.

Previous clinical studies demonstrated optimistic results regarding the safety and potential efficacy of various types of SC administration into the corpus cavernosum for the treatment of ED patients with different pathophysiologies [24-28]. Most published human studies used MSCs in patients who had undergone radical prostatectomy (RP) $[26,27]$. The pathophysiology of ED post$\mathrm{RP}$ is quite different from that in diabetic patients: physical injury to the neurovascular bundle is the principal pathogenesis of ED after RP. This may ultimately result in corporeal fibrosis and veno-occlusive dysfunction [29]. ED in diabetic patients is mainly a functional disorder resulting from impaired NO production by endothelial cells [30]. Therefore, we included only diabetic patients with ED in our trial as different pathophysiologies may result in a different response to this treatment.

Yiou et al. [26] studied post-prostatectomy patients who were administered an escalating number of bone marrow mononuclear cells in stage I and II studies. The authors reported single IC of autologous BM-MNC to be safe and effective in ED patients after RP followed up for 12 months. Likewise, Haahr et al. [27] used autologous adipose-derived regenerative cells in patients with ED after RP. The authors observed a positive effect of SC injection only in patients without urinary incontinence. This leaves the possibility of psychological elements deterring patients with incontinence from improved sexual performance.

Bahk et al. [28] published the first study that addressed SCs in diabetic patients with ED. The investigators used a single IC injection of allogeneic umbilical cord blood SCs and reported positive effects on both ED and DM; however, their study did not investigate the safety of IC SCs injections. Furthermore, the use of allogeneic umbilical cord blood SCs results in a small total number of SCs that can be injected. As stated by the authors, this may lead to an insufficient improvement in sexual function. In our study, we used 2 IC injections of allogeneic WJ-MSCs which usually results in a higher number of SCs that are injected.

Recently, Bieri et al. [25] found that IC injection of nonexpanded bone marrow concentrate for the treatment of ED was safe and effective. However, the efficacy was demonstrated based only on improvements in the IIEF-5 score, as no significant change was seen on Doppler ultrasound. Similarly, our group [24] in a pilot clinical trial studied the safety and efficacy of 2 consecutive IC injections of allogeneic bone marrow-MSCs in 4 diabetic patients with ED. We found that the procedure was welltolerated and safe, and significantly improved overall sexual function scores. Again, the efficacy measurement tools in this pilot study were subjective. In this current study, we demonstrated the efficacy based on significant changes in IIEF-5 and EHS together with significant improvements in penile hemodynamics as demonstrated by CDDU.

Regarding the safety of IC injection of SCs, there were no serious adverse events reported by any of the previous clinical studies. Thus, our study results are in accordance with the previously published clinical trials. Haahr et al. [27] reported that from 21 patients who were injected with autologous adipose-derived regenerative cells, 8 men experienced transient redness and swelling at the injection site. One patient developed an abdominal hematoma at the fat harvest site, which led to scrotal and penile hematoma. Furthermore, Bieri et al. [25] reported that no long-term adverse events were noted related to the intervention except for minimal and short-term bruising at the site of harvest or injection.

In summary, we evaluated the safety and efficacy of 2 consecutive IC injections of WJ-MSCs for the treatment of diabetic patients with ED for 12 months. The safety and tolerability were demonstrated clinically and by extensive laboratory examinations. The efficacy of the treatment was evaluated subjectively by validated questionnaires and objectively by CDDU. Thus, this treatment was found to be tolerable and safe, and also effective in improving $\mathrm{EF}$ in diabetic patients with ED. The decline of the positive effects seen between months 6 and 12 of follow-up may indicate that the improvement in EF is limited in duration.

Our study has several limitations. This study was unblinded and without a control group. The small number of patients recruited in this study was principally driven by the low social acceptance of this new, as yet unproven, treatment modality. However, as we have demonstrated 
the safety and efficacy of this treatment, we are recruiting a large number of patients with prolonged follow-up periods. Further studies for dose-finding and studies with double-blinding and a control group will have to be undertaken in order to further assess this treatment for ED in diabetic patients.

\section{Conclusion}

This is the first human clinical trial on diabetic patients with ED that used 2 consecutive IC injections of allogeneic WJ-MSCs. The current findings of this study support the safety and efficacy profile of IC injections of allogeneic WJ-MSCs to treat diabetic patients with ED.

\section{Statement of Ethics}

The study was approved by the Institutional Review Board of Cell Therapy Center, the University of Jordan, Amman, Jordan (reference no. IRB/4/2017), and prospectively registered on clinicaltrials.gov (NCT02945449). All participants were informed re- garding the study. Participants signed a written informed consent before participation in the study following the Helsinki Declaration.

\section{Conflict of Interest Statement}

The authors have no conflicts of interest to declare.

\section{Funding Sources}

This study was funded by the Deanship of Scientific Research/ the University of Jordan.

\section{Author Contributions}

1. Saddam Al Demour: manuscript writing, data collection, conceived and designed the analysis, and design of the research.

2. Sofia Adwan: data collection and manuscript writing.

3. Hanan Jafar: analysis of the results and design of the research.

4. Reem Rahmeh: data collection and analysis of the results.

5. Hussam Alhawari: data collection and manuscript writing.

6. Abdalla Awidi: main conceptual idea and design of the research.

\section{References}

1 Korneyev IA, Alexeeva TA, Al-Shukri SH, Bernikov AN, Erkovich AA, Kamalov AA, et al. Prevalence and risk factors for erectile dysfunction and lower urinary tract symptoms in Russian Federation men: analysis from a national population-based multicenter study. Int J Impot Res. 2016 Mar-Apr;28(2):74-9.

2 Gerber RE, Vita JA, Ganz P, Wager CG, Araujo AB, Rosen RC, et al. Association of peripheral microvascular dysfunction and erectile dysfunction. J Urol. 2015;193(2):612-7.

3 Divers J, Mayer-Davis EJ, Lawrence JM, Isom S, Dabelea D, Dolan L, et al. Trends in incidence of type 1 and type 2 diabetes among youths: selected counties and Indian reservations, United States, 2002-2015. MMWR Morb Mortal Wkly Rep. 2020;69(6):161-5.

4 Corona G, Giorda CB, Cucinotta D, Guida P, Nada E, Aglialoro A, et al. Sexual dysfunction in type 2 diabetes at diagnosis: progression over time and drug and non-drug correlated factors. PLoS One. 2016;11(10):e0157915.

5 Mazzilli R, Elia J, Delfino M, Benedetti F, Scordovillo G, Mazzilli F. Prevalence of diabetes mellitus (DM) in a population of men affected by Erectile Dysfunction (ED). Clin Ter. 2015;166(5):e317.

6 Porst H, Burnett A, Brock G, Ghanem H, Giuliano F, Glina S, et al. SOP conservative (medical and mechanical) treatment of erectile dysfunction. J Sex Med. 2013;10(1):13071.
7 Alwaal A, Zaid UB, Lin CS, Lue TF. Stem cell treatment of erectile dysfunction. Adv Drug Deliv Rev. 2015;82-83:137-44.

8 Dashwood M R, Crump A, Shi-Wen X, Loesch A. Identification of neuronal nitric oxide synthase (nNOS) in human penis: a potential role of reduced neuronally-derived nitric oxide in erectile dysfunction. Curr Pharm Biotechnol. 2011;12(9):1316-21.

9 Zhou F, Xin H, Liu T, Li GY, Gao ZZ, Liu J, et al. Effects of icariside II on improving erectile function in rats with streptozotocin-induced diabetes. J Androl. 2012 Sep-Oct;33(5):83244.

10 Stuckey BGA, Jadzinsky MN, Murphy LJ, Montorsi F, Kadioglu A, Fraige F, et al. Sildenafil citrate for treatment of erectile dysfunction in men with type 1 diabetes: Results of a randomized controlled trial. Diabetes Care. 2003;26(2):279-84.

11 Wang X, Liu C, Xu Y, Chen P, Shen Y, Xu Y, et al. Combination of mesenchymal stem cell injection with icariin for the treatment of diabetes-associated erectile dysfunction. PLoS One. 2017;12(3):1-18.

12 Gokce A, Peak TC, Abdel-Mageed AB, Hellstrom WJ. Adipose tissue-derived stem cells for the treatment of erectile dysfunction. Curr Urol Rep. 2016;17(2):1-7.

13 Ryu JK, Kim DH, Song KM, Ryu DS, Kim SN, Shin $\mathrm{DH}$, et al. Intracavernous delivery of clonal mesenchymal stem cells rescues erec- tile function in the streptozotocin-induced diabetic mouse. Andrology. 2016;4(1):17284.

14 Albersen M, Kendirci M, van der Aa F, Hellstrom WJ, Lue TF, Spees JL. Multipotent stromal cell therapy for cavernous nerve injuryinduced erectile dysfunction. J Sex Med. 2012; 9(2):385-403.

15 Wang HS, Hung SC, Peng ST, Huang CC Wei HM, Guo YJ, et al. Mesenchymal stem cells in the Wharton's jelly of the human umbilical cord. Stem Cells. 2004;22(7): 1330-7.

16 Karahuseyinoglu S, Cinar O, Kilic E, Kara F, Akay GG, Demiralp DO, et al. Biology of stem cells in human umbilical cord stroma: in situ and in vitro surveys. Stem Cells. 2007 Feb; 25(2):319-31.

17 Fong CY, Richards M, Manasi N, Biswas A, Bongso A. Comparative growth behaviour and characterization of stem cells from human Wharton's jelly. Reprod Biomed Online. 2007;15(6):708-18

18 Pittenger MF, Mackay AM, Beck SC, Jaiswal RK, Douglas R, Mosca JD, et al. Multilineage potential of adult human mesenchymal stem cells. Science. 1999;284(5411):143-7.

19 Abediankenari S, Ghasemi M. Generation of immune inhibitory dendritic cells and $\mathrm{CD} 4+\mathrm{T}$ regulatory cells inducing by TGF- $\beta$. Iran J Allergy Asthma Immunol. 2009;8(1): 25-30. 
20 Haahr MK, Jensen $\mathrm{CH}$, Toyserkani NM, Andersen DC, Damkier P, Sørensen JA, et al. Safety and potential effect of a single intracavernous injection of autologous adipose-derived regenerative cells in patients with erectile dysfunction following radical prostatectomy: an open-label phase I clinical trial. EBioMedicine. 2016;5:20410.

21 Levy JA, Marchand M, Iorio L, Cassini W, Zahalsky MP. Determining the feasibility of managing erectile dysfunction in humans with placental-derived stem cells. J Am Osteopath Assoc. 2016;116(1):e1-5.

22 Sarugaser R, Lickorish D, Baksh D, Hosseini MM, Davies JE. Human umbilical cord perivascular (HUCPV) cells: a source of mesenchymal progenitors. Stem Cells. 2005;23(2): 220-9.

23 Margossian T, Reppel L, Makdissy N, Stoltz JF, Bensoussan D, Huselstein C. Mesenchymal stem cells derived from Wharton's jelly: comparative phenotype analysis between tis- sue and in vitro expansion. Biomed Mater Eng. 2012;22(4):243-54.

24 Al Demour S, Jafar H, Adwan S, Alsharif A, Alhawari H, Alrabadi A, et al. Safety and potential therapeutic effect of two intracavernous autologous bone marrow derived mesenchymal stem cells injections in diabetic patients with erectile dysfunction: an open label phase I clinical trial. Urol Int. 2018;101(3): 358.

25 Bieri M, Said E, Antonini G, Dickerson D, Tuma J, Bartlett CE, et al. Phase I and registry study of autologous bone marrow concentrate evaluated in PDE5 inhibitor refractory erectile dysfunction. J Transl Med. 2020; 18(1):24-7.

26 Yiou R, Hamidou L, Birebent B, Bitari D, Le Corvoisier P, Contremoulins I, et al. Intracavernous injections of bone marrow mononucleated cells for postradical prostatectomy erectile dysfunction: final results of the INSTIN clinical trial. Eur Urol Focus. 2017;3(6): 643-5.
27 Haahr MK, Harken Jensen C, Toyserkani NM, Andersen DC, Damkier P, Sørensen JA, et al. A 12-month follow-up after a single intracavernous injection of autologous adipose-derived regenerative cells in patients with erectile dysfunction following radical prostatectomy: an open-label phase I clinical trial. Urology. 2018;121:203.e613.

28 Bahk JY, Jung JH, Han H, Min SK, Lee YS. Treatment of diabetic impotence with umbilical cord blood stem cell intracavernosal transplant: preliminary report of 7 cases. Exp Clin Transplant. 2010;8(2):150.

29 Leungwattanakij S, Bivalacqua TJ, Usta MF, Yang DY, Hyun JS, Champion HC, et al. Cavernous neurotomy causes hypoxia and fibrosis in rat corpus cavernosum. J Androl. 2003 Mar-Apr;24(2):239-45.

30 Ning H, Qiu X, Baine L, Lin G, Lue TF, Lin CS. Effects of high glucose on human cavernous endothelial cells. Urology. 2012;80(5): 1162-11. 